\title{
6 TRANSPARENCIA EN LA GESTIÓN DE LAS BIBLIOTECAS PÚBLICAS DEL ESTADO A TRAVES DE SUS SEDES WEB
}

\section{Management transparency in state-run public libraries: Website information}

\author{
Ana-Reyes Pacios, Blanca Rodríguez-Bravo, Marina Vianello-Osti, Carina \\ Rey-Martín y Concepción Rodríguez-Parada
}
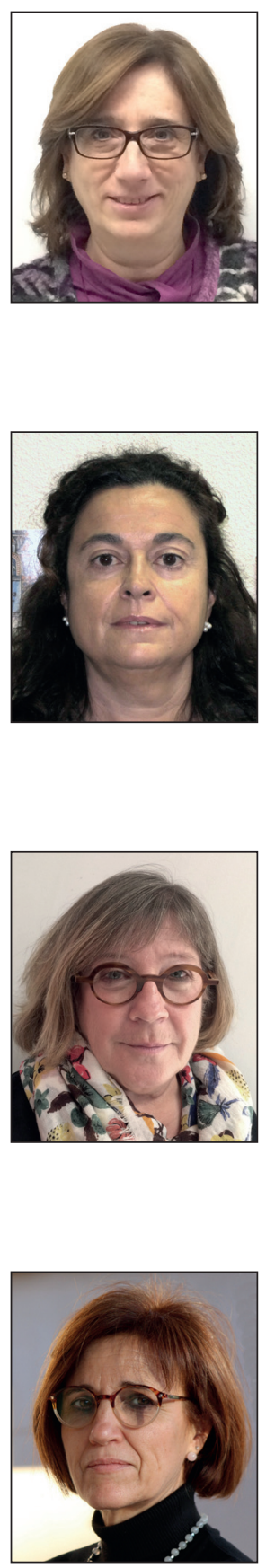

Ana-Reyes Pacios, doctora en Filosofía y Letras por la Universidad de León, es profesora titular del Departamento de Biblioteconomía y Documentación de la Universidad Carlos III de Madrid (UC3M) desde 1992. Imparte clases sobre gestión y dirección de bibliotecas en los estudios de grado, master y doctorado. Su principal línea de investigación son las funciones y técnicas de gestión aplicadas a las bibliotecas (evaluación, calidad, planificación y marketing) y las fuentes de información. Autora y co-autora de trabajos publicados en revistas nacionales e internacionales del área (Library management, Library review, Libri, The electronic library, etc.). http://orcid.org/0000-0003-2887-882X

Universidad Carlos III de Madrid, Departamento de Biblioteconomía y Documentación Madrid, 126-128. 28903 Getafe (Madrid), España areyes@bib.uc3m.es

Blanca Rodríguez-Bravo es profesora titular de universidad en el Área de Biblioteconomía y Documentación en la Universidad de León. Sus intereses científicos se dirigen al estudio de la utilización de revistas electrónicas, el desarrollo de colecciones, la comunicación científica y la organización del conocimiento. Ha dirigido proyectos de investigación y ha publicado trabajos relacionados con estos campos en libros, revistas científicas y actas de congresos.

http://orcid.org/0000-0002-9476-7602

Universidad de León, Facultad de Filosofía y Letras Departamento de Patrimonio Artístico y Documental, Área de Biblioteconomía y Documentación Campus de Vegazana, s/n. 24071 León, España blanca.rodriguez@unileon.es

Marina Vianello-Osti es licenciada en Lettere italiane por la Università Ca' Foscari di Venezia, en filología por la Universidad Complutense de Madrid y doctora en documentación por la Universidad Carlos III de Madrid (UC3M). Ha sido responsable del centro de documentación del Instituto de Demografía del CSIC. Desde 1996 pertenece al Departamento de Biblioteconomía y Documentación de la UC3M donde da clases de historia, análisis de documentos, y descripción del libro antiguo. Su investigación se centra en la historia de la alfabetización, organización del conocimiento, análisis de contenido, hipertexto y usabilidad.

http://orcid.org/0000-0002-8745-3947

Universidad Carlos III de Madrid, Departamento de Biblioteconomía y Documentación Madrid, 126-128. 28903 Getafe (Madrid), España mvianell@bib.uc3m.es

Carina Rey-Martín, doctora por la Universidad Complutense de Madrid, es profesora titular de universidad del Departamento de Biblioteconomía, Documentación y Comunicación Audiovisual de la Universitat de Barcelona. Imparte clases sobre gestión y dirección de bibliotecas en los estudios de grado y master. Sus principales líneas de investigación son las técnicas de gestión aplicadas a las bibliotecas (evaluación, calidad, planificación y marketing), la modelización para la toma de decisiones y el desarrollo de los servicios bibliotecarios.

http://orcid.org/0000-0002-5238-7353

Universitat de Barcelona, Facultat de Biblioteconomia i Documentació Departamento de Biblioteconomía, Documentación y Comunicación Audiovisual Melcior de Palau, 140. 08014 Barcelona, España carina.rey@ub.edu 


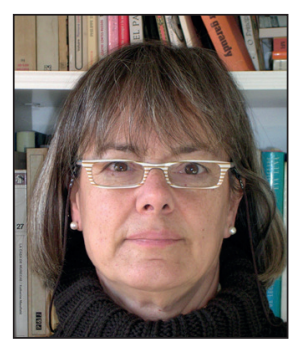

Concepción Rodríguez-Parada, licenciada en Filosofía, diplomada en Biblioteconomía y Documentación y doctora por la Universidad de Barcelona (UB), es profesora titular del Departamento de Biblioteconomía, Documentación y Comunicación Audiovisual de la UB. Coordina el Master de Gestión y Dirección de Bibliotecas y Servicios de Información. Miembro de la comisión redactora del Codi deontològic del Cobdc y del Grupo de Trabajo de Ética Profesional redactor del Código deontólogico de Sedic, adoptado por Fesabid en 2013. Sus líneas de investigación se centran en la gestión bibliotecaria, el patrimonio bibliográfico y la espiritualidad femenina medieval. http://orcid.org/0000-0001-8161-7579

Universitat de Barcelona, Facultat de Biblioteconomia i Documentació Departamento de Biblioteconomía, Documentación y Comunicación Audiovisua Melcior de Palau, 140. 08014 Barcelona, España crodriguezp@ub.edu

\section{Resumen}

Partiendo de una propuesta de indicadores asociados a la transparencia en la gestión de bibliotecas, basada en ejemplos localizados en sus sedes web, se presentan los resultados del análisis en las 53 bibliotecas públicas del Estado. La búsqueda y análisis de los indicadores se llevó a cabo en sus webs. Los resultados muestran que, aunque hay ejemplos de buenas prácticas, la información al respecto es muy escasa, especialmente la relacionada con las líneas de actuación y objetivos de la biblioteca. La normativa sobre el uso de los servicios, así como la información y contacto con el equipo de profesionales de la biblioteca, son dos de los indicadores más frecuentes. Se recomienda a las bibliotecas, como entidades del sector público receptoras de fondos y subvenciones, que hagan un ejercicio de transparencia publicando los indicadores relacionados con la misma en su sede web.

\section{Palabras clave}

Bibliotecas públicas; Bibliotecas públicas del Estado; Transparencia; Indicadores; Gestión; Gestión de bibliotecas; Webs; España.

\section{Abstract}

This article discusses the results of analysing management transparency in 53 Spanish's state-run libraries based on a proposed series of indicators drawn from the libraries' own websites. While the findings detected examples of good practice, information was observed to be scant, particularly in terms of libraries' lines of action and objectives. The rules and procedures for using the services and contacting with librarians were the types of information most frequently provided. The authors recommend that libraries, as public institutions receiving funds and subsidies from the government, should engage voluntarily in transparency by publishing information on their indicators on their websites.

\section{Keywords}

Public libraries; State-run libraries; Transparency; Indicators; Management; Libraries management; Web sites; Spain.

Pacios, Ana-Reyes; Rodríguez-Bravo, Blanca; Vianello-Osti, Marina; Rey-Martín, Carina; Rodríguez-Parada, Concepción (2018). "Transparencia en la gestión de las bibliotecas públicas del Estado a través de sus sedes web". El profesional de la información, v. 27, n. 1, pp. 36-48.

https://doi.org/10.3145/epi.2018.ene.04

\section{Introducción}

La Ley 19/2013, de 9 de diciembre, de transparencia, acceso a la información pública y buen gobierno (España, 2013) (en adelante, Ltaipbg) obliga a las administraciones públicas estatales, regionales y locales a presentar información relevante para garantizar el funcionamiento y el control de la actividad pública. Desde su entrada en vigor, a partir de diciembre de 2014, ha movido a algunas instituciones a crear portales de transparencia para ir demostrando su aplicación. A la vez, ha llevado a otras a evaluar su grado de cumplimiento en distintos ámbitos, como por ejemplo la Fundación Compromiso y Transparencia, que, en su empeño por impulsar la transparencia, el buen gobierno y la responsabilidad social corporativa, elabora una serie de informes que analizan la información publicada en los portales web de diversos grupos de entidades de interés público (ej. universidades, museos, partidos políticos, etc.). Se evalúa, en especial, la información a la que obliga la Ley relacionada con la publicidad activa y el acceso a la información pública, enunciadas en los arts. 5 a 24. Hasta el momento, los informes relativos a su aplicación revelan que las instituciones y organismos analizados, obligados por ésta, tienen unos niveles notables y sobresalientes en materia de transparencia. Además, se concluye que la transparencia que practican no es sólo cuantitativa, sino también cualitativa, logrando muy altos niveles de calidad (Consejo de Transparencia y Buen Gobierno, 2017, p. 11).

Como consecuencia de la aplicación de la Ltaipbg, así como de los trabajos dedicados a su evaluación (Moreno-Sardà; Molina-Rodríguez-Navas; Simelio-Solà, 2017), se está creando una cultura de transparencia en las instituciones. A la vez, el concepto de transparencia aumenta día a día su valor y está siendo una oportunidad para algunas instituciones que se han hecho más responsables con ella. Posiblemente tengan en cuenta que, cuanto mayor sea la transparencia, mayor será la confianza de los ciudadanos en ellas. 
Las bibliotecas, como instituciones culturales receptoras de fondos públicos, también pueden involucrarse en la transparencia, aunque no estuvieran obligadas por la Ley por depender de una institución o entidad mayor y estar obligada ésta, y mostrarse más abiertas ante los ciudadanos que utilizan sus servicios, proporcionando información sobre cómo se gestionan. Puede ser una oportunidad para generar confianza, dando información suficiente sobre lo que hacen y cómo lo hacen, a la vez que rinden cuentas e incitan a la participación de los ciudadanos. Algunas, como la biblioteca de la UNED, demuestran haber asumido la cultura de la transparencia creando un portal con esa denominación (Biblioteca transparente) en el que agrupan, estructuran y dan acceso a los datos e información relacionados con su gestión.

En este trabajo se pretende conocer si las bibliotecas públicas del Estado (en adelante, BPE) se han sumado a la aplicación de la Ltaipbg, como sujetos obligados, según el art. 2, apartado 1, letra a), si utilizan su web con esta finalidad y a qué tipo de información sobre la gestión están dando acceso. En particular, la indicada en el Capítulo II dedicado a la Publicidad activa.

\section{Transparencia y bibliotecas}

Las definiciones de transparencia, analizadas por Oztoprak y Ruijer (2016, p. 536), comparten y evolucionan en torno a una comprensión común del término: poner a disposición de cualquiera la información institucional. Pero, como señala el mismo Oztoprak, la transparencia se extiende más allá de la apertura. Una organización puede abrir sus documentos, operaciones y resultados, simplemente publicándolos, sin embargo, no puede definirse como transparente si la información disponible no puede entenderse y utilizarse activamente por los interesados.

Se podría calificar de escasa y más bien reciente la bibliografía relativa a la transparencia referida a las bibliotecas, si la búsqueda de estudios se realiza utilizando este término. Sin embargo, el interés por conocer si las bibliotecas dan a conocer datos relacionados con su gestión no es nuevo. Hay una serie de estudios que persiguieron este objetivo poniendo de relieve que a medida que pasaba el tiempo los documentos que tenían que ver con la gestión se fueron incrementando, caso de las universitarias (Pacios, 2003). También en éstas se observó este mismo comportamiento con ciertos documentos (ej. proyectos, políticas y procedimientos, directorio de personal) al analizar la evolución de las webs en una muestra de bibliotecas (Aharony, 2011, p. 770). El trabajo de Burke (2016) analiza una serie de items ligados a la transparencia en las bibliotecas públicas y su evolución en el tiempo, mostrando, al igual que los anteriores, su incremento significativo entre los años 2012 y 2015. Sin duda las webs no sólo se están utilizando para promover las visitas, dar visibilidad a las bibliotecas y los servicios que ofrecen, también se están poniendo al servicio de la transparencia.

La transparencia en las bibliotecas se ha venido relacionando, fundamentalmente, con la elaboración de memorias o informes anuales, al mostrar las decisiones que toman los responsables de su gestión (Carmena-Escribano, 1993). Por otra parte, se ha demostrado que una memoria es una buena herramienta al servicio de las relaciones públicas de la biblioteca en la medida en que contribuye a mejorar su percepción (Sauer, 2006, p. 21; Lear, 2006, p. 462). Según Wilson y Goolsby, (2002) es perfecta para compartir los proyectos y objetivos de la biblioteca con todos los que la apoyan.

Trabajos más recientes vinculan la transparencia con la apertura sin condiciones a determinado tipo de información. Es el caso de Johnson (2011, p. 98) que recomienda los pasos a seguir para que los bibliotecarios escolares desarrollen una cultura de transparencia y generen confianza entre los usuarios, abriendo algunas "ventanas" en las que se publique:

- el presupuesto, además de invitar al proceso de su elaboración;

- el calendario que permita ver lo que se hace en el tiempo; - los objetivos, de forma que se puedan comentar y discutir; - las estadísticas, de modo que los que las vean puedan hacer preguntas.

Indica además que debe existir una cultura de puertas abiertas, para que cualquiera tenga la oportunidad de ver cómo se trabaja, "que las paredes sean transparentes". Y también mostrar y estar abierto a las opiniones. Las partes interesadas en la biblioteca pueden estar de acuerdo o en desacuerdo, pero deben conocer las creencias fundamentales sobre la misma.

En España algunos profesionales de las bibliotecas han dado un toque de atención para sumarse a la transparencia haciendo uso de determinados documentos, como las cartas de servicio, por constituir éstas un compromiso con los ciudadanos (García-Maza, 2003). Más recientemente García-Arribas (2015, p. 44-45) estima la conveniencia de que la biblioteca pública publicite activamente, sin que se reciba requerimiento alguno, los siguientes documentos y datos, de acuerdo con la Ltaipbg: líneas estratégicas de la gestión y datos que apoyen y evalúen la gestión bibliotecaria. Éste autor señala que deben

"hacerlos comprensibles, es decir, permitir el acceso a los documentos que dan contexto a esos datos y que crean información elaborada sobre la conveniencia de determinados servicios..." (García-Arribas, 2015, p. 45).

En la línea de estas propuestas que apuestan por una cultura de transparencia en las bibliotecas, mostrando determinado tipo de información, datos y documentos concretos, se realizó una propuesta de indicadores para las bibliotecas universitarias que fue aplicada a las universitarias del consorcio Madroño (Pacios, 2016).

En suma, transparencia y rendición de cuentas en las bibliotecas son temas cuyo interés aumenta a juzgar por los trabajos que van surgiendo. Es importante que las propuestas sirvan para que las bibliotecas conozcan su nivel de transparencia, con el fin de poder compararse con otras similares y mejorar su nivel efectivo.

\section{La gestión de las bibliotecas públicas del Estado (BPE)}

Las 53 BPE que son objeto de análisis en este trabajo tienen la peculiaridad de ser de titularidad estatal pero su gestión 
está transferida al organismo autónomo al que pertenecen (Diputación, Consejería de Cultura) desde los años 80. Sólo una, la BPE de Vitoria tiene la gestión transferida a un organismo de administración local, la Diputación Foral de Álava (Hernández-Sánchez, 2001, p. 221). Tal y como indica el art. 149.1.28 de la Constitución española:

"El Estado tiene competencia exclusiva sobre las bibliotecas de titularidad estatal, sin perjuicio de su gestión por parte de las comunidades autónomas".

Respecto a su consideración como centros culturales, tal y como se indica en la web del Ministerio de Educación, Cultura y Deporte (en adelante, $M E C D$ ), son:

"centros de especial relevancia para el desarrollo cultural del país y el acceso a la información de los ciudadanos. Son, por lo general, las bibliotecas más importantes de las ciudades en las que se ubican y su presencia en el panorama de la actividad bibliotecaria nacional está reconocida" (MECD, 2015).

Sin duda son candidatas a mostrarse transparentes.

Una visión panorámica de las BPE, a través de los datos globales que se muestran en la web del MECD de los 5 últimos años, que también se confirman en el Anuario de estadísticas culturales (MECD, 2016, p. 278), permite afirmar que los usuarios inscritos aumentaron un 0,14\%, aunque los visitantes disminuyeron un $0,11 \%$, así como los prestatarios activos que se redujeron un $0,22 \%$. La impresión que transmiten estos datos es que las bibliotecas están viendo disminuir sus usuarios activos y han aumentado considerablemente las actividades que realizan, posiblemente ante la demanda de los ciudadanos o para captar usuarios. La interpretación de esta información puede ser tan subjetiva y dar una imagen tan deformada de la realidad que las propias bibliotecas deberían evitar que fuera así, aportando otros datos que permitieran conocer su situación real. No en vano hay trabajos que han pretendido destacar el valor económico y social de las bibliotecas porque suelen pasar desapercibidos (Gómez-Yáñez, 2014; Togores-Martínez, 2015; Hernández-Sánchez, 2016), así como la necesidad de contar con herramientas que recojan el retorno social y económico de su actividad (Carrión-Gútiez, 2013).

También se han presentado ejemplos y casos de éxito relacionados con su gestión, basados en procedimientos como la planificación estratégica, el trabajo en colaboración, la complicidad de los usuarios, la búsqueda de vías alternativas de financiación, la creatividad, la imaginación, la inventiva, la experimentación y la innovación como opciones de garantía que permiten reforzar la utilidad social de las bibliotecas en su comunidad (García-Gómez, 2015). Está claro que los responsables de las bibliotecas públicas, en foros en los que tienen cabida como profesionales, manifiestan lo que hacen y cómo lo hacen y hay ejemplos excepcionales de su modo de trabajar. Sin embargo, estos trabajos no llegan a los usuarios ni a otras personas interesadas en conocer cómo se gestionan y lo que están aportando a la sociedad. Por eso puede ser una buena táctica facilitar esta información desde la web de la biblioteca para que cualquier ciudadano pueda consultarla.

Por otra parte, al igual que ocurre en otros países, las biblio- tecas están fuertemente condicionadas por los procesos políticos y las decisiones que se toman en consecuencia (Jaeger; Bertot; Gorham, 2013, p. 61). Ante esta realidad resulta de interés aportar datos sobre su gestión con el fin de poder defender mejor sus intereses y necesidades, así como articular mejor sus posiciones ante el público. Cuando los datos son precisos, presentados de manera objetiva y utilizados para la mejora, y con la intención de rendir cuentas al exterior, sirven para equilibrar la presión y el apoyo. En España, la inestabilidad y desigualdad que ocasionan los vaivenes políticos, unido a las circunstancias de crisis o abundancia, han llevado a denunciar y reclamar una política de Estado en el ámbito de las bibliotecas públicas que las convierta en una prioridad política (Sánchez-Sánchez, 2017a). Iniciativas como la llevada a cabo en su día por la Fundación Bertelsmann (García; Sutherland, 2001) en la que se reunió en un mismo foro a políticos y directores de bibliotecas podrían permitir acercar posiciones entre unos y otros.

Acerca de la gestión de estas bibliotecas apenas se encuentran trabajos relacionados. Los localizados se refieren a las bibliotecas públicas municipales (Arroyo-Ortega, 2004; García-Rodríguez, 2005, Soto-Aranzadi, 2006; Urkia-Etxabe, 2008). Si acudimos a las leyes de bibliotecas con las que cuentan las comunidades autónomas a las que corresponde su gestión no queda claro quién es el titular de la misma (órgano, director u otro) y cómo se lleva a cabo. En algunas, por ejemplo las de Galicia y Aragón, se hace mención a un órgano administrativo de dirección y coordinación que depende de la Consejería de Cultura y es el encargado de:

"establecer anualmente en sus presupuestos programas para la adquisición de fondos y recursos tecnológicos, actividades de difusión y formación de personal y otros que pudiesen considerarse necesarios" (Galicia, 2012, art. 7; España, 2012).

En otras, se hace referencia a comisiones asesoras y consejos técnicos que intervienen en la gestión de las bibliotecas (ej. Castilla la Mancha, 2011, art. 34; España, 2011). Juan Sánchez-Sánchez define bien la situación de las bibliotecas españolas a través de la legislación:

"Las distintas leyes de bibliotecas de comunidades autónomas pueden servir de ejemplo del mayor o menor interés hacia el servicio bibliotecario y permiten constatar que en España no tenemos una política bibliotecaria de Estado sino algo que se parece a un mosaico con 17 políticas bibliotecarias distintas" (Sánchez-Sánchez, 2017b).

Situados en la web del MECD donde hay enlaces a cada biblioteca pública del Estado se ve el organismo del que depende:

- 51 bibliotecas lo hacen del gobierno de la comunidad autónoma correspondiente (13 de una dirección general, 6 de una subdirección general, 31 de un servicio y 1 directamente de la consejería);

- 1 de un consejo insular (de una dirección);

- 1 de la diputación (de un departamento).

http://mapabpe. $m$ cu.es/mapabpe.cmd?command=GetMapa

La situación es muy variopinta, lo que hace suponer que afectará al grado de autonomía de su gestión por parte del responsable de la biblioteca. Todas cuentan con un director. 
Acerca de esta figura, o responsable de la biblioteca y las funciones que desempeña, la consulta de los perfiles profesionales bibliotecarios del Sistema Bibliotecario Español, con sus correspondientes competencias, tiene como ámbito específico o áreas de trabajo en las que desarrollan sus tareas,

"la gestión, supervisión y coordinación de las diferentes áreas del centro" (Tejadas-Artigas; Martínez-González, 2013, p. 8).

Entre las funciones propias del perfil están las relacionadas con (Tejadas-Artigas; Martínez-González, 2013, p. 8-9):

- planificar la política del servicio;

- dirigir y supervisar proyectos;

- dirigir la planificación estratégica;

- gestionar los recursos económicos;

- supervisar sistemas de gestión de la calidad;

- dirigir el equipo humano;

- dirigir la información y comunicación del centro;

- evaluar los servicios;

- coordinar procesos y servicios, etc.

Algunos de los resultados de esta actividad gestora son los que convendría poner en la web de la biblioteca, demostrando así transparencia en la gestión que realizan.

Dadas las particularidades de cada comunidad autónoma, surgen dudas al leer la legislación de cada una acerca de si entre las funciones del responsable está la gestión del presupuesto, lo que demostraría su capacidad de decisión y autonomía. Puestos en contacto con algunos de los responsables de estas bibliotecas para conocer esta particularidad, se podría afirmar que no todos los directores de las BPE tienen capacidad de ejecución presupuestaria, más allá de la que afecta a la selección de las adquisiciones o de las actividades de extensión bibliotecaria o cultural. Aunque se dan distintos casos por la situación administrativa de la biblioteca en relación con la comunidad autónoma de la que depende, hay dos circunstancias derivadas de si las bibliotecas tienen o no la consideración de "unidad gestora del gasto":

- Las que están en comunidades autónomas pluriprovinciales, que dependen, orgánica y presupuestariamente, de las delegaciones provinciales (DP), que son las que tienen la consideración de "unidad gestora del gasto", adjudican a los directores de las bibliotecas la responsabilidad de selección de los fondos bibliográficos. Pero el límite presupuestario se lo marcan desde las DP. Estas comunidades tienen, además, una biblioteca cabecera del sistema y, en determinados casos, la biblioteca pública del Estado y la autonómica comparten el mismo edificio, por ejemplo, en Andalucía y Castilla La Mancha.

- Las que están en comunidades autónomas uniprovinciales adoptan varias soluciones. Unas, como la de Cantabria, tienen identificadas las funciones de la biblioteca autonómica con las de la pública del Estado en una misma estructura. Otras, como la de Asturias las tiene diferenciadas. Y pueden tener o no la categoría de "unidad gestora del gasto" dependiendo de la consideración que le otorgue el organismo autónomo. Por ejemplo, Murcia y La Rioja la tienen, lo que implica que cuentan con un programa presupuestario propio.
Esta situación revela las diferencias entre unas bibliotecas y otras, según su consideración como "unidad de gasto" o no, sus funciones atribuidas, su tamaño y el organismo autonómico del que dependen, lo que sin duda influye decisivamente en la capacidad de toma de decisiones en la gestión.

\section{Metodología}

Para la búsqueda de información relacionada con la transparencia se partió de una plantilla de indicadores asociados a la misma propuesta para las bibliotecas universitarias ( $\mathrm{Pa}$ cios, 2016). Este modelo se modificó según las particularidades de las bibliotecas públicas, reduciendo el número de indicadores a 17. Se tuvo también en cuenta el tipo de documentos considerados en otros trabajos para examinar la transparencia en las bibliotecas públicas (Burke, 2016, p. 451), así como la información sujeta a publicación que establece la Ltaipbg, en su art. II dedicado a la publicidad activa, y cuya concreción se refleja en la Metodología de evaluación y seguimiento de la transparencia de la actividad pública (Mesta) (Aeval, 2016) pero que requiere de una adaptación para el caso particular de las bibliotecas. Estos 17 indicadores están ligados a áreas de información relevante (tabla 1). Se entiende aquí el indicador como la unidad de información (factor, variable o característica específica que se muestra en forma de documento, dato o símbolo) observable y medible para apreciar un logro, cambio o desempeño a efectos de transparencia. Se consideró para su definición los posibles interesados en esta información:

- instituciones u organismos que pudieran contribuir a su financiación,

- políticos,

- beneficiarios de sus servicios,

- equipo de profesionales que trabaja en la biblioteca,

- otros profesionales interesados en realizar comparaciones para contrastar datos,

- bibliotecas con las que se coopera y trabaja,

- además de los usuarios o ciudadanos interesados en conocer lo que hacen y cómo lo hacen.

Una vez considerada la validez de los indicadores para las bibliotecas públicas, la búsqueda y análisis de los mismos en el grupo de las 53 BPE se llevó a cabo en junio de 2017. Los pasos seguidos fueron los siguientes:

- Revisión de las webs que cada biblioteca tiene en el MECD y que responden a un mismo modelo, con el mismo tipo de información, aunque particular de cada una (en adelante, primera web).

- Revisión de la web de cada biblioteca (en adelante, segunda web), a partir del enlace ubicado en cada una de las páginas que tienen en el portal del MECD. Es en esta web en la que se ha centrado la búsqueda de los indicadores sobre transparencia porque desde ésta se informa y da acceso a los diferentes servicios.

- Recogida de la información en una hoja Excel para visualizar los resultados y extraer datos totales relacionados con la presencia de los indicadores en las bibliotecas.

Para estimar idónea esta información de las primeras webs de las bibliotecas se aplicó una serie de criterios considerados en otros trabajos que analizan la transparencia y que sirvieron de modelo para llevar a cabo éste (Martín-Cavan- 
Tabla 1. Indicadores de transparencia agrupados por áreas informativas relevantes

\begin{tabular}{|c|c|}
\hline Áreas informativas & Indicadores \\
\hline \multirow{2}{*}{ 1. Propósito del servicio y objetivos que persigue } & A) Definición de la misión \\
\hline & B) Plan estratégico \\
\hline \multirow{4}{*}{ 2. Órganos de gobierno y reglas de funcionamiento } & C) Miembros que integran las comisiones relacionadas con la gestión de la biblioteca \\
\hline & D) Reglamento \\
\hline & E) Normativa específica de funcionamiento de los servicios \\
\hline & F) Carta de deberes y derechos de los usuarios \\
\hline 3. Oferta de servicios & G) Carta de servicios \\
\hline 4. Recursos de información & H) Política/programa de gestión de la colección \\
\hline \multirow{2}{*}{ 5. Personal } & I) Organigrama \\
\hline & J) Directorio de personal \\
\hline \multirow{4}{*}{ 6. Resultados } & K) Indicadores de gestión (Cuadro de mando) \\
\hline & L) Encuestas de satisfacción \\
\hline & M) Memoria anual o informe de actividades \\
\hline & N) Reconocimientos, premios, certificaciones \\
\hline \multirow{2}{*}{ 7. Información económica } & O) Presupuesto \\
\hline & P) Contratos de licitación y concursos \\
\hline 8. Pertenencia a redes y otras relaciones de colaboración & Q) Redes con las que se trabaja y coopera \\
\hline
\end{tabular}

na; Barrio, 2016). En particular, se consideró conveniente tener en cuenta los siguientes, apoyándose además en los principios de Norman (1998) y los del diseño de interacción de Tognazzini (2014), también presentes en la metodología Mesta ya referida:

- visibilidad: la información es fácilmente accesible y situada en un lugar claramente perceptible;

- accesibilidad: la información no necesita de permisos para ser consultada;

- actualidad: en el caso de esta información se consideró que debía responder al año 2016 por ser el año anterior al que se realizó el trabajo;

- comprensibilidad: la información se presenta escrita en las lenguas oficiales del país;

- integralidad: la información debe ser completa y exhaustiva;

- a estos criterios se une además, de acuerdo con la Ltaipbg (art. 11), la reutilización, es decir, la información debe estar en un formato que pueda ser utilizada por cualquier persona física o jurídica.

\section{Resultados y discusión}

En primer lugar, en relación con la revisión de cada web albergada en el MECD (primera web), se observa que el ciudadano o el profesional que se plantee conseguir información de las BPE no encuentra con facilidad un portal o un listado para acceder a ellas. En esta web no hay un enlace que nos remita directamente a esta información. Se han tenido que visitar diferentes páginas con denominaciones ambiguas para llegar a la información que se buscaba (ej. Publicaciones y Bibliotecas). Finalmente, en Cultura $>$ Bibliotecas $>\mathrm{Pa}$ norámica de las $\mathrm{BPE}$, se llega a la información de cada una de las páginas de las BPE, por lo que la ruta a seguir para llegar al listado no cumple con los criterios de visibilidad.

Desde la página "Panorámica de las 53 BPE", se accede a información recabada de las bibliotecas y elaborada por el
$M E C D$ que se organiza en secciones. Aunque no existe una sección dedicada de forma explícita a la transparencia, tanto la sección "Mapa", el "Anexo estadístico" como "Evolución" recogen información referida al período 2011-15, susceptible de ser objeto de una política de transparencia que es de interés para este análisis en cuanto aparecen datos que responden a indicadores establecidos, como el presupuesto (gastos e inversiones de cada biblioteca). Sin embargo, esta información no está en un formato reutilizable.

\section{La localización de los documentos que responden a los indicadores está disper- sa en diferentes enlaces}

En la sección "Mapa” se localizan unos enlaces con el nombre de la ciudad en la que se ubica cada BPE que reenvía a la web de cada una. Todas están elaboradas siguiendo los mismos criterios de organización de los contenidos. El diseño de esta información resulta coherente gracias a que en el marco superior aparece el nombre del portal y el logotipo del $M E C D$, evitando así que el usuario dude acerca de la autoría de la información.

http://mapabpe.mcu.es/mapabpe.cmd?command=GetMapa

Una vez se accede a cada una de las webs (segunda), a través del enlace disponible en el $M E C D$, el panorama es el siguiente:

- 17 bibliotecas cuentan con una web de diseño consistente que permite identificarlas como miembros de la red webs, aunque presentan variaciones en la organización de sus contenidos;

- el resto, cuenta con una web que, por pertenecer a la red de la comunidad autónoma, sigue las normas de estilo de la misma, aunque también se encuentran variantes en los enlaces que contienen. 
Esta falta de homogeneidad en esta segunda web de las BPE, nos ha hecho desistir de aplicar un análisis sistemático a estos sitios considerando que requerirían un estudio aparte. Por otra parte, la dispersión de los documentos que responden a los indicadores de transparencia en estas webs hace difícil realizar una comparativa.

En lo referente al análisis de los indicadores localizados en la sede web de cada biblioteca (segunda web), en la tabla 2 se recogen los resultados globales, en forma de porcentaje. Y, a continuación, se comentan las peculiaridades que presentan en algunas bibliotecas, poniendo ejemplos de aquellas buenas prácticas observadas.

La localización de los documentos que responden a los indicadores está dispersa en diferentes enlaces. Hay uno que suele recoger, en ciertos casos, la mayoría. Aparece bajo las denominaciones de "La biblioteca", "Sobre la biblioteca", "Mi biblioteca" o "Tu biblioteca". Bajo este enlace se pueden agrupar otros, como el titulado "Quiénes somos", "Gestión" en el que se encuentran algunos de los documentos. Otra página que contiene documentos es la dedicada a los servicios, en la que se pueden hallar, por ejemplo, las normativas de uso de los mismos.

Tabla 2. Presencia de los indicadores en las sedes web de las BPE

\begin{tabular}{|l|c|}
\hline \multicolumn{1}{|c|}{ Indicadores } & $\begin{array}{c}\text { Presencia en la web } \\
\text { de las bibliotecas } \\
\text { (\%) }\end{array}$ \\
\hline A) Definición de la misión & 39,62 \\
\hline B) Plan estratégico & 7,55 \\
\hline $\begin{array}{l}\text { C) Miembros que integran las comisiones que participan en } \\
\text { la gestión de la biblioteca }\end{array}$ & 1,89 \\
\hline D) Reglamento (Normas generales de uso de la biblioteca) & 56,60 \\
\hline E) Normativa específica de funcionamiento de los servicios & 86,79 \\
\hline F) Carta de deberes y derechos de los usuarios & 0 \\
\hline G) Carta de servicios & 39,62 \\
\hline H) Política/programa de gestión de la colección & 1,89 \\
\hline I) Organigrama & 0 \\
\hline J) Directorio de personal & 62,26 \\
\hline K) Indicadores de gestión (Cuadro de mando) & 1,89 \\
\hline L) Encuestas de satisfacción & 7,55 \\
\hline M) Memoria anual o informe de actividades & 7,55 \\
\hline N) Reconocimientos, premios, certificaciones & 3,77 \\
\hline O) Presupuesto & 3,77 \\
\hline P) Contratos de licitación y concursos & 0,57 \\
\hline Q) Redes con las que se trabaja y coopera & \begin{tabular}{c} 
l, \\
\hline
\end{tabular} \\
\hline
\end{tabular}

\section{A) Misión}

Representa el propósito o la razón de ser de la biblioteca y aparece en 21 casos (39,6\%). Es una declaración que al definirse en un proceso de planificación suele ser habitual que aparezca junto a la visión y los valores de la biblioteca. Se localizaron casos ejemplares como las bibliotecas de Lugo, Toledo y Tarragona, que la muestran dentro del enlace titulado "Quiénes somos" y "La biblioteca". Encontramos bibliotecas que han definido la misión al elaborar la carta de servicios, por lo que se encuentra dentro de este documento. Se da también el caso de aquellas bibliotecas que utilizan la definición de la misión que la IFLA atribuye a las bibliotecas públicas, la cual no se asocia a un proceso de reflexión como conviene en este tipo de manifestación escrita sobre lo que quiere lograr y a dónde quiere llegar la institución (Evans; Alire, 2013, p. 89).

\section{B) Plan estratégico}

Es uno de los documentos más importantes ya que muestra el camino que pretende seguir la biblioteca y su orientación hacia objetivos. Sin embargo, tiene una escasa presencia en el conjunto de webs con sólo 4 ejemplos (7,5\%). Se trata de los casos de Gerona, Lérida, Tarragona y Toledo. Algunas bibliotecas muestran objetivos generales aunque no responden a un tiempo concreto de consecución.

\section{C) Miembros que integran las comisiones relacionadas con la gestión de la biblio- teca}

Las leyes de bibliotecas de las comunidades autónomas a las que pertenecen las biblio- 
tecas hacen mención, en su mayoría, a un órgano colegiado (generalmente con la denominación de comisión o consejo) que desempeña funciones de tipo consultivo y asesor (ejemplo de Aragón, Cantabria). También se encuentran, en otros casos, referencias a comisiones técnicas que intervienen en la dirección y coordinación de las bibliotecas que pertenecen a la misma red (caso de Castilla La Mancha). En este sentido, aunque estas comisiones sean de ámbito autonómico, si de algún modo participan en la gestión de la biblioteca o toman decisiones relacionadas con la misma, se debería conocer el órgano, los miembros que lo componen y las funciones que realiza. Sólo en el caso de la Biblioteca de Zamora, localizamos dentro de su equipo el Centro Provincial Coordinador de Bibliotecas con el nombre de sus integrantes. En el resto no se ha localizado ninguna alusión al respecto.

\section{D) Reglamento o normativa de uso de la biblioteca}

En la mayoría de los localizados, este documento consiste en una sencilla guía de uso de la biblioteca en la que se fijan normas mínimas de comportamiento que debe cumplir el usuario con las correspondientes sanciones en caso de que no sea así, o también, una serie de puntos relativos al funcionamiento correcto de los servicios. Puede tratarse también de una página web en la que, en forma de FAQs, se va dando respuesta a la normativa de acceso a la biblioteca. En la contabilización total se han considerado estos casos, llegando a estar presente en 30 bibliotecas (56,60\%). Hay bibliotecas que, por pertenecer a una misma comunidad autónoma, comparten este documento como es el caso de las de Andalucía.

Los documentos aparecen bajo las denominaciones de "La biblioteca", "Sobre la biblioteca", "Mi biblioteca" o "Tu biblioteca"

\section{E) Normativa específica de funcionamiento de los servicios}

Es el documento más habitual, como también es obvio, destinado a que los usuarios conozcan las reglas de funcionamiento de los servicios que presta la biblioteca, de ahí que esté presente en 46 (86,79\%). En este momento es muy corriente encontrar las condiciones de uso de tablets, acceso a internet, salas de trabajo, etc., y, por supuesto, las relacionadas con el préstamo que son las que tienen mayor presencia en el conjunto. Bibliotecas de la misma comunidad autónoma comparten algunos de estos documentos, como el reglamento de préstamo interbibliotecario en las de Castilla la Mancha.

\section{F) Carta de deberes y derechos de los usuarios}

No hay ninguna biblioteca que tenga un documento titulado de este modo. Sin embargo, hay que hacer la observación de que aquellas bibliotecas que cuentan con una carta de servicios tienen un apartado dedicado a los derechos de los usuarios.

\section{G) Carta de servicios}

Ésta supone un compromiso de calidad de la biblioteca con sus usuarios. Además de los servicios recoge una serie de obligaciones a las que se compromete la biblioteca que suponen un valor añadido para los usuarios y cuyo debido cumplimiento puede ser exigido en todo momento por los mismos a través de los canales de comunicación que se establecen. Se encuentra presente en 21 bibliotecas (39,62\%). Constituye una buena práctica en materia de evaluación de la calidad de la biblioteca al introducir las unidades de medida o indicadores para verificar el grado de cumplimiento de estos compromisos, siempre y cuando se haga un seguimiento de los mismos. Algunas, por pertenecer a la misma comunidad autónoma como las andaluzas, responden a un mismo modelo.

\section{H) Política o programa de gestión de la colección}

Este documento sirve para justificar ante el usuario las prioridades que tiene la biblioteca en materia de gestión de la colección y a qué criterios obedecen. Además, orienta al personal de la biblioteca a la hora de las adquisiciones y favorece la cooperación (CCB, 2010). Las recomendaciones al 
respecto de los expertos (Clayton; Gorman, 2006) indican que la política debería incluir el reparto del presupuesto según prioridades y materias. Es, por tanto, un documento que requiere de una actualización constante. Sólo se ha localizado una política $(1,89 \%)$ en la muestra de bibliotecas que corresponde a la Biblioteca de Tarragona.

\section{l) Organigrama}

Se entiende éste como la representación gráfica de la estructura de la biblioteca, en la que se muestran las relaciones entre sus departamentos o partes y la función de cada una de ellas, así como de las personas que trabajan en las mismas. No existe ninguna biblioteca que disponga del mismo. Bien es verdad que muchas bibliotecas dan a conocer el equipo de profesionales con el que cuentan siguiendo un cierto orden jerárquico, atendiendo a su categoría profesional, pero no se trata en realidad de un organigrama como tal.

\section{J) Directorio de personal}

Los localizados responden a un listado o relación de nombres de las personas que trabajan en la biblioteca. No se suele utilizar esta denominación, es más habitual la de "Equipo", aunque hay alguna excepción como la de Toledo. Aparecen incluidas en los servicios de los que forman parte, en su mayoría con su correo-e y el teléfono de contacto. No existe la posibilidad de búsqueda por servicio o persona. En esta situación se localizó un total de 33 (62,26\%), siendo esta información una de las que tiene mayor presencia en el conjunto, aspecto coincidente con el trabajo de Burke (2016, p. 456).

La carta de servicios constituye una buena práctica en materia de evaluación de la biblioteca... siempre y cuando se haga un seguimiento de los indicadores para verificar el cumplimiento de los compromisos

\section{K) Indicadores de gestión (Cuadro de mando)}

Nos referimos aquí al conjunto de indicadores que la biblioteca elige para medir, generalmente de forma anual, su rendimiento. Son importantes para conocer cómo evolucionan los diferentes ámbitos de análisis, además de permitir la comparación con otras similares o con aquellas que podrían ser consideradas el referente a seguir. Posiblemente el ejemplo más significativo en las públicas haya sido en su momento el conjunto de indicadores del programa PAB (Programa de análisis de bibliotecas) que impulsó la Fundación Bertelsmann en el año 1993 que tuvo su continuidad en otros proyectos hasta 2007 (Espinàs, 2001; Felis, 2004, p. 11). Sólo se ha localizado 1 biblioteca (1,89\%) que muestre algo similar: Santiago de Compostela. Cuenta con 41 indicadores rela-

cionados con el equipamiento, recursos humanos, recursos informativos, oferta y uso de servicios, que se pueden ver en su balance anual, en particular el de 2015. Compara sus valores con la media española, de modo que permite ver su situación en relación con este valor, resultando un balance positivo. Hay que tener en cuenta que en la Comunidad de Galicia, las estadísticas de las bibliotecas incluyen una serie de indicadores que permiten a cada biblioteca hacer un seguimiento de su situación y compararse con el resto. Desde la página web de cada una titulada "Estadísticas, informes y memorias" se puede acceder a esta información.

\section{Las estadísticas no resultan comprensi-} bles si no se acompañan de explicaciones o valores de referencia que añadan valor a las cifras

También hay que advertir que las bibliotecas que tienen una carta de servicios cuentan con una serie de indicadores de los que se presupone su seguimiento en un período temporal concreto. Así queda demostrado en el caso de la Biblioteca de Toledo, que presenta datos relativos a la revisión de los compromisos desde 2013 a 2016.

\section{L) Encuestas de satisfacción}

Permiten conocer la opinión del usuario en relación con los servicios que presta la biblioteca y, por tanto, aportan información sobre su calidad y las posibilidades de mejora. La medición periódica de su punto de vista demuestra el interés por conocer también las expectativas y los cambios que se producen conforme pasa el tiempo. Dar a conocer estos datos es un indicador de transparencia. Las bibliotecas con cartas de servicios suelen incluir, entre sus compromisos, la realización de encuestas de satisfacción. Hay bibliotecas que en el período de búsqueda de los indicadores para la realización de este trabajo contaban con una encuesta de opinión dirigida a los usuarios, caso de Jaén, sobre el préstamo de e-books. 
http://www.juntadeandalucia.es/cultura/opencms/export/ download/bibjaen/ENCUESTA_de_opinixn_e-Readers.pdf

En el conjunto de las BPE objeto de análisis son 4 (7,55\%) las que muestran los resultados de las encuestas de satisfacción: Murcia, Toledo, Gerona y Tarragona. Esta última tiene una serie muy completa, desde el año 1999 hasta el 2016, que permite ver la evolución de la opinión de sus usuarios a lo largo del tiempo.

\section{M) Memoria anual o informe de actividades}

Este documento aporta información sobre las actividades realizadas por la biblioteca durante el año, a modo de balance. Suele incluir los datos de la ejecución del presupuesto, estadísticas, indicadores, resultados de encuestas, etc. Las localizadas en esta muestra son 4 (7,55\%): Mahón, Huelva, Santiago de Compostela y Almería. La extensión y el grado de detalle varía mucho de unas a otras. Por ejemplo, la de Mahón es muy exhaustiva y extensa, y permite conocer los puntos débiles y amenazas de la biblioteca, como la carencia de personal, equipamiento inadecuado, etc. La de Santiago, bajo el título "Balance del año 2015", ofrece un documento sintético a modo de presentación, que se ha considerado en este apartado teniendo en cuenta su adecuado contenido.

La información que facilitan las BPE en su web es muy escasa, salvo excepciones, especialmente la que tiene que ver con la planificación y evaluación

\section{N) Reconocimientos, premios, certificaciones}

Se trata de información positiva para la biblioteca en la medida en que demuestra cómo está haciendo las cosas y que evidencia algún aspecto relacionado con la calidad de la misma. Son 2 las BPE $(3,77 \%)$, las de Melilla y Toledo, que muestran algún reconocimiento de este tipo, relacionado con la certificación de la calidad de los servicios. La de MeliIla, según consta en su web,

“obtuvo el Certificado de Registro de Empresa con el número ER-1359/2006 que evidencia la conformidad de su Servicio de Gestión de Calidad con los requisitos de la Norma UNE-EN ISO 9001:2000".

La de Toledo tiene la carta de servicios certificada por Aenor desde 2008.

\section{0) Presupuesto}

Sólo 2 bibliotecas $(3,77 \%)$ dan a conocer el presupuesto y su ejecución. La de Santiago de Compostela lo incluye en el balance que tiene a modo de memoria de actividades y resultados. En el caso de la de Mahón, lo encontramos también en uno de los apartados de la memoria. Otras bibliotecas, para temas presupuestarios, remiten a los datos que aparecen en la web que tiene cada una de estas bibliotecas en el $M E C D$, por lo que quedaría cubierta la información de naturaleza económica, presupuestaria y estadística a la que se refiere la Ltaipbg, en su artículo 7, pero se precisa visitar otra web diferente. No hay que olvidar que en este modelo uniforme de sitio todas cuentan con los mismos enlaces en los que se accede a datos relativos a los gastos, tanto los corrientes como de inversión. Por otra parte, también encontramos bibliotecas que, además, remiten enlazando a las estadísticas de la comunidad autónoma a la que pertenecen, por ejemplo las de Cataluña y Galicia.

\section{P) Contratos de licitación y concursos}

No se ha encontrado referencia alguna a esta información que permite conocer detalles de los contratos de licitación para la adquisición de lotes de libros, licencias de uso de libros electrónicos, contratación de servicios, etc. Algunas convocatorias de contratos públicos relacionados con estas $\mathrm{BPE}$, caso de inversiones en la colección, los edificios y determinados equipamientos, se pueden ver en la página de contratación del Ministerio.

\section{Q) Redes con las que se trabaja y coopera}

La mayoría de las bibliotecas, 44 (90,57\%) presentan en su web el icono o logo de las redes en las que se integran y con las que cooperan. Las más comunes son tres:

- la de la comunidad autónoma a la que pertenece la biblioteca;

- e-biblio (Cuadrado-Fernández; Fe-Trillo, 2015): proyecto iniciado en 2014, impulsado por la Secretaría de Estado de Cultura en colaboración con las comunidades autónomas que ofrece un servicio gratuito y online de préstamo de libros electrónicos;

- la Red de sedes web: otro proyecto del Ministerio que ha permitido a las bibliotecas públicas diseñar sus propias webs.

Planes e indicadores de seguimiento de los resultados tienen una presencia testimonial

\section{Conclusiones y recomendaciones}

Una vez realizado el análisis y expuestos los resultados se llega a las siguientes conclusiones:

1) El presente trabajo no pone en duda que las BPE cuenten con muchos de los documentos e información ligados a cada uno de los indicadores establecidos de transparencia. Sin embargo, se demuestra aquí que no utilizan sus sedes web para darlos a conocer. Los usuarios tienen derecho a acceder a esta información y puede existir interés público en conocerla.

2) EI MECD hace un ejercicio de transparencia poniendo a disposición información estadística de las 53 bibliotecas. Sin embargo, para llegar dentro de este portal al lugar donde se encuentra la información de las BPE, la ruta a seguir no cumple con los criterios de visibilidad por no ser clara la organización del sitio y tener que dar excesivos pasos en la ruta. Todas las secciones analizadas de este portal son accesibles sin necesidad de registro alguno y en castellano. Pero no existe la posibilidad de seleccionar ninguna de las otras lenguas del territorio nacional. Las páginas son coherentes pero la navegabilidad es mejorable en alguna de sus secciones (ej. el enlace "Evolución") por la ausencia de un 
sistema (migas de pan) que permita recordar la secuencia de los apartados y verificar en qué lugar nos encontramos, teniendo que volver a la primera página de la sección. Por otra parte, el estilo de la presentación y la presencia constante del logotipo el MECD en el marco superior garantiza la coherencia de las páginas, evitando la desorientación de los usuarios. La información proporcionada llega hasta el año 2015. En cuanto a la integralidad, teniendo en cuenta los indicadores de transparencia establecidos, los datos estadísticos posibilitan conocer su evolución a largo de un período, desde 2011 a 2015, así como la comparativa entre unas y otras, pero serían convenientes otros datos que, puestos en relación con los facilitados, permitieran hacer una valoración inicial de su rendimiento (ISO, 2014). Las estadísticas, aunque ofrecen datos significativos de la biblioteca, no resultan comprensibles si no se acompañan de explicaciones o valores de referencia que añadan valor a las cifras y, por otra parte, esta información no es reutilizable.

3) Aunque no contamos con unos estándares de transparencia para las bibliotecas, teniendo en cuenta los indicadores propuestos asociados a la gestión, se puede afirmar que la información al respecto que facilitan las BPE en sus webs es muy escasa, salvo excepciones, especialmente la que tiene que ver con la planificación y evaluación de la misma. Planes e indicadores de seguimiento de los resultados tienen una presencia testimonial en el conjunto.

4) Sólo 4 de los 17 indicadores establecidos superan el 50\% de presencia en las webs. Los contenidos mejor representados se relacionan con las redes de las que forman parte las $B P E$, la información y los datos de contacto del equipo de profesionales de la biblioteca y la normativa específica de uso de los servicios.

5) Los ausentes del conjunto son las cartas de deberes y derechos de los usuarios, el organigrama y los contratos de licitación y concursos. Aunque hay que considerar que los derechos de los usuarios quedan recogidos en las cartas de servicio con las que cuentan algunas bibliotecas (39,62\%). Uno de los documentos peor representados, que sorprende por su importancia para la biblioteca, es la política o programa de gestión de la colección, a pesar de que el Ministerio ha dado pautas para su elaboración.

http://travesia.mcu.es/portalnb/jspui/bitstream/10421/5081/1/ GT_Sel_cooperativa.pdf

6) El presupuesto ejecutado de la biblioteca debería mostrarse con detalle de las partidas para que resulte comprensible y fiable. Sería conveniente que, aunque no lo gestione la biblioteca, el capítulo relativo al gasto de personal se diera a conocer de forma que se pueda calcular el coste total anual de la misma. Para conocerlo hay que ir a la página web de la biblioteca con sede en el Ministerio.

7) Las BPE que se muestran como ejemplo de buenas prácticas son las de Tarragona, Toledo y Santiago de Compostela, teniendo en cuenta el tipo y número de documentos puestos a disposición de cualquier usuario y/o ciudadano.

La Ltaipbg y la bibliografía surgida al respecto promueven la transparencia de las instituciones públicas. Las sedes web son un instrumento esencial para acercar la información a los ciudadanos. Las BPE, aunque no estuvieran obligadas a cumplir la Ley, como entidades del sector público receptoras de fondos y subvenciones, pueden hacer un ejercicio de transparencia voluntaria poniendo a disposición en su sede web los indicadores sobre la gestión que se proponen en este trabajo. No se sabe el efecto que puede tener hacerlo, pero estaría de sobra justificado si se tiene en cuenta que, tanto el Manifiesto de la IFLA sobre Transparencia, buen gobierno y erradicación de la corrupción (IFLA, 2008) como el Código de ética de la profesión (IFLA, 2013), consideran a los bibliotecarios esenciales para favorecer el acceso a la información, para una ciudadanía bien informada y un gobierno transparente. Si, además, la IFLA también aboga por la biblioteca como proveedora de información, sería obvio asumir que la transparencia de su propia gestión va a ser apoyada por los bibliotecarios responsables de la misma (Burke, 2016, p. 464).

La propuesta presentada en este trabajo, adaptación de la Ltaipbg a una biblioteca, y su aplicación a esta muestra de BPE, permite tener una aproximación a este tipo de información puesta a disposición y puede ser un primer paso para acercarnos a la transparencia de las bibliotecas públicas. También hay que tener en cuenta que la propuesta está basada en los ejemplos localizados de bibliotecas que ya están realizando este ejercicio de transparencia voluntaria.

\section{Agradecimientos}

Nuestro agradecimiento a todos los directores de las bibliotecas con los que nos pusimos en contacto por responder a las preguntas. En especial a José M. Gutiérrez-Rodríguez por las sugerencias aportadas a este trabajo.

\section{Referencias}

Aeval (2016). Metodología de evaluación y seguimiento de la transparencia de la actividad pública. Agencia Estatal de Evaluación de las Políticas Públicas y la Calidad de los Servicios.

http://www.aeval.es/es/difusion_y_comunicacion/publicaciones/Informes/Informes_de_Evaluacion/Evaluaciones_2016/E42.html

Aharony, Noa (2011). "An analysis of American academic libraries' websites: 2000-2010". The electronic library, v. 30, n. 6, pp. 764-776.

https://goo.gl/cCXzmD

https://doi.org/10.1108/02640471211282091

Arroyo-Ortega, Óscar (2004). "El modelo Castilla-La Mancha de financiación para la adquisición de fondos documentales en bibliotecas públicas municipales". En: /l Congreso nacional de bibliotecas públicas: La biblioteca pública, compromiso de futuro. Salamanca, pp. 23- 31.

http://travesia.mcu.es/portalnb/jspui/bitstream/10421/711/1/ CongresoNacionalBP_02.pdf

Burke, Susan K. (2016). "Public library administration: Transparency on the website". The library quarterly, v. 86, n. 4, pp. 449-467.

https://doi.org/10.1086/688033

Carmena-Escribano, Miguel A. (1993). "Tomar decisiones en una biblioteca. Algunas sugerencias sobre las posibles pautas a seguir en la elaboración de memorias - informes". 
Boletín de la Asociación Andaluza de Bibliotecarios, n. 33, pp. 25-40.

Carrión-Gútiez, Alejandro (2013). “Informe de situación de las bibliotecas públicas españolas en 2012". El profesional de la información, v. 22, n. 3, pp. 250-258.

https://doi.org/10.3145/epi.2013.may.09

Castilla-La Mancha (2011). "Ley 3/2011, de 24 de febrero, de la Lectura y de las Bibliotecas de Castilla-La Mancha". DOCM, n. 46, 8 de marzo.

http://docm.castillalamancha.es/portaldocm/descargarArchivo. do? ruta=2011/03/08/pdf/2011_3585.pdf\&tipo=rutaDocm

CCB (2010). Pautas para establecer una política de colecciones en una biblioteca pública. Consejo de Cooperación Bibliotecaria. Grupo de Trabajo de Selección Bibliográfica Cooperativa.

http://travesia.mcu.es/portalnb/jspui/bitstream/10421/5081/1/ GT_Sel_cooperativa.pdf

Clayton, Peter; Gorman, G. E. (2006). Managing information resources in libraries: collection management in theory and practice. London: Facet Publishing. ISBN: 9781856045810

Consejo de Transparencia y Buen Gobierno (2017). Informe de evaluación del cumplimiento de la Ley de Transparencia. http://www.consejodetransparencia.es/dam/jcr:a8fc69aee37d-4961-8fd7-8077274240ea/Resumen-ejecutivo-MESTA_ def_webmejorado.pdf

Cuadrado-Fernández, María-Isabel; Fe-Trillo, María-José (2015). "eBiblio, servicio de préstamo de libros electrónicos en bibliotecas públicas". El profesional de la información, v. 24, n. 2, pp. 176-184.

https://doi.org/10.3145/epi.2015.mar.11

España (2011). "Ley 3/2011, de 24 de febrero, de la Lectura y de las Bibliotecas de Castilla-La Mancha". BOE, n. 103, 30 de abril.

https://www.boe.es/boe/dias/2011/04/30/pdfs/BOE-A-2011-7708. $p d f$

España (2012). "Ley 5/2012, de 15 de junio, de bibliotecas de Galicia". BOE, n. 161, 6 de julio.

https://www.boe.es/buscar/pdf/2012/BOE-A-2012-9061-consolidado. $p d f$

España (2013). “Ley 19/2013, de 9 de diciembre, de transparencia, acceso a la información pública y buen gobierno". $B O E$, n. 295, 10 de diciembre.

https://www.boe.es/buscar/doc.php?id=BOE-A-2013-12887

Espinàs, Eulàlia (2001). "El programa de análisis de bibliotecas (PAB)". En: VIII Octavas jornadas catalanas de documentación. Barcelona: Col·legi Oficial de Bibliotecaris-Documentalistes de Catalunya.

http://www.cobdc.org/jornades/8JCD/comunicacions/05_02comunic. $p d f$

Evans, G. Edward; Alire, Camila (2013). Management basics for information professionals. American Library Association. ISBN: 9781555709099

Felis, Agnés (2004). "El programa de análisis de bibliotecas de la Fundación Bertelsmann dará paso en 2005 al 'foro $\mathrm{PAB}^{\prime \prime}$. Anaquel: Boletín de libros, archivos y bibliotecas de
Castilla-La Mancha, n. 27, p. 11.

http://ccta.jccm.es/public_dglab/aplicaciones/archivos/ anaquel_pdfs/200501282102339106.pdf

Galicia (2012). "Ley 5/2012, de 15 de junio, de bibliotecas de Galicia”. DOG, n. 122, 27 de junio.

https://goo.gl/ysjRTz

García, June; Sutherland, Sue (2001). Directores de la biblioteca pública en la arena política. Barcelona: Fundación Bertelsmann. ISBN: 849321551

https://www.fundacionbertelsmann.org/es/home/ publicaciones-raiz/publicacion/did/directores-de-la-bibliotecapublica-en-la-arena-politica

García-Arribas, Roberto (2015). "Transparencia y participación de los ciudadanos en las bibliotecas públicas: hacia una nueva coproducción de servicios entre los ciudadanos y bibliotecarios". En: VII Congreso nacional de bibliotecas públicas. Bibliotecas públicas, conectados contigo. Madrid: Ministerio de Cultura, pp. 43-51.

http://travesia.mcu.es/portalnb/jspui/bitstream/10421/8988/3/ VIICNBP_l.pdf

García-Gómez, Francisco-Javier (2015). “Gestión bibliotecaria contra la recesión: Creatividad, imaginación e innovación". En: VII Congreso nacional de bibliotecas públicas. Bibliotecas públicas, conectados contigo. Madrid: Ministerio de Cultura, p. 139-147.

http://travesia.mcu.es/portalnb/jspui/bitstream/10421/8988/3/ VIICNBP_l.pdf

García-Maza, Julia (2003). “Exigencia de transparencia vs. vocación de opacidad: función social de las cartas de servicios en las bibliotecas". En: VIII Jornadas españolas de documentación. Fesabid, Barcelona, pp. 109-118.

García-Rodríguez, Araceli (2006). "Modelos de gestión de bibliotecas municipales en España". En: I Encuentro bibliotecas y municipio. La administración local y las bibliotecas en la democracia. Peñaranda de Bracamonte, 2005. Madrid: Ministerio de Cultura, pp. 25-39 o 135-142.

http://travesia.mcu.es/portalnb/jspui/handle/10421/1328

Gómez-Yáñez, José-Antonio (coord.) (2014). El valor económico y social de los servicios de información: bibliotecas. Madrid: Ministerio de Educación, Cultura y Deporte, Fesabid. http://www.fesabid.org/sites/default/files/repositorio/fesabidvalor-economico-social-servicios-informacion-bibliotecas.pdf

Hernández-Sánchez, Hilario (2001). "Red de bibliotecas y gestión territorial". En: Cultura, desarrollo y territorio. III Jornadas iniciativa privada y sector público en la gestión de la cultura. Vitoria: Xabide, pp. 215-226. ISBN: 9788492127449

Hernández-Sánchez, Hilario (coord.) (2016). Estudio de impacto socioeconómico de las bibliotecas en la Comunidad Foral de Navarra. El valor de las bibliotecas. Pamplona: Gobierno de Navarra.

http://www.ccbiblio.es/wp-content/uploads/info_elvalor Navarra.pdf

IFLA (2008). Manifiesto de la IFLA sobre Transparencia, buen gobierno y erradicación de la corrupción.

https://www.ifla.org/ES/publications/manifiesto-de-la-ifla-sobretransparencia--buen-gobierno-y-erradicaci-n-de-la-corrupci-n 
IFLA (2013). Código de ética de los bibliotecarios y profesionales de la información en España.

https://www.ifla.org/files/assets/faife/codesofethics/spain-x

ISO (2014). Standard 11620:2014. Information and documentation - Library performance indicators.

Jaeger, Paul T.; Bertot, John-Carlo; Gorham, Ursula (2013). "Wake up the nation: Public libraries, policy making, and political discourse". The library quarterly, v. 83, n. 1, pp. 61-72. https://goo.gl/qdphpA https://doi.org/10.1086/668582

Johnson, Doug (2012). "Transparency and trust". Library media connection, v. 30, n. 6, p. 98.

http://www.doug-johnson.com/dougwri/transparencyand-trust.html

Lear, Bernadette A. (2006). "'Tis better to be brief than tedious?'. The evolution of the American Public Library Annual Report, 1876-2004". Libraries \& the cultural record, v. 41, n. 4, pp. 462-486

https://doi.org/10.1353/lac.2006.0060

Martín-Cavanna, Javier; Barrio, Esther (2016). Examen de transparencia. Informe de transparencia voluntaria en la web de las universidades españolas 2015. Madrid: Fundación Compromiso y Transparencia.

MECD (2015). Panorámica de las 53 bibliotecas públicas del Estado.

http://mapabpe.mcu.es/mapabpe.cmd?command=GetPortada

MECD (2016). Anuario de estadísticas culturales 2016. Subdirección General de Estadística y Estudios, Secretaría General Técnica. https://goo.gl/gfWtp2

Moreno-Sardà, Amparo; Molina-Rodríguez-Navas, Pedro; Simelio-Solà, Núria (2017). "Impacto de la legislación sobre transparencia en la información publicada por las administraciones locales". El profesional de la información, v. 26, n. 3, pp. 370-380.

https://doi.org/10.3145/epi.2017.may.03

Norman, Donald A. (1998). The design of everyday things. London: MIT. ISBN: 0385267746

http://wireframe.vn/books/Design\%200f\%20Everyday\%20 Things.pdf

Oztoprak, Abdullah-Abid; Ruijer, Erna (2015). "Variants of transparency: an analysis of the English local government transparency code 2015". Local government studies, v. 42, n. 4, pp. 536-556.

https://goo.gl/aQ5zZ4

https://doi.org/10.1080/03003930.2016.1154845

Pacios, Ana-Reyes (2003). "Management-related information on Spanish university library webpages". The electronic library, v. 21, n. 6, p. 528-537.

https://doi.org/10.1108/02640470310509081

Pacios, Ana-Reyes (2016). "Universidades transparentes con bibliotecas transparentes". Investigación bibliotecológica, v. 30, n. 70, pp. 105-128.

https://doi.org/10.1016/j.ibbai.2016.10.006

Sánchez-Sánchez, Juan (2017a). Elogio de la biblioteca pública. Buenos Aires: Alfagrama. ISBN: 9789871305964

Sánchez-Sánchez, Juan (2017b). “El 'cuarto mundo' de los servicios bibliotecarios en España”. Foro IweTel, 11 de julio. https://goo.gl/KfhHWM

Sauer, James (2006). "The annual report. The academic librarian's tool for management, strategic planning and advocacy". College \& undergraduate libraries, v. 13, n. 2, pp. 21-34.

https://doi.org/10.1300/J106v13n02_02

Soto-Aranzadi, Susana (2006). "Modelos de gestión de bibliotecas municipales: la experiencia de la red municipal de las bibliotecas de Donostia San Sebastián". En: I Encuentro de bibliotecas y municipio (1. Peñaranda de Bracamonte, Salamanca, 2005) Madrid: Ministerio de Cultura, Secretaría Técnica, pp. 135-142.

http://travesia.mcu.es/portalnb/jspui/handle/10421/1347

Tejada-Artigas, Carlos; Martínez-González, Belén (coords.) (2013). Perfiles profesionales del Sistema Bibliotecario Español: fichas de caracterización. Ministerio de Educación, Cultura y Deporte; Consejo de Cooperación Bibliotecaria.

http://travesia.mcu.es/portalnb/jspui/bitstream/10421/6841/1/ perfilesprofesionalesSBE.pdf

Tognazzini, Bruce (2014). "First principles of interaction design". AskTOG. Interaction design solutions for the real world, 5 March.

http://asktog.com/atc/principles-of-interaction-design

Togores-Martínez, Rosa (2015). El valor de les biblioteques públiques en la societat: el cas de la Xarxa de Biblioteques Municipals. Barcelona: Diputació de Barcelona. Gabinet de Premsa i Comunicació.

https://goo.gl/s2CyNd

Urkia-Etxabe, Aranxa (2008). "La Red de bibliotecas municipales de Donostia San Sebastián año 2008. Su modelo de gestión, sus proyectos cooperativos". En: IV Congreso nacional de bibliotecas públicas. A Coruña.

http://travesia.mcu.es/portalnb/jspui/bitstream/10421/534/1/ com_199.pdf

Wilson, John S.; Goolsby, Mary (2002). "The value of an annual report in library development". The bottom line, v. 15, n. 2, pp. 91-93.

https://doi.org/10.1108/bl.2002.17015bab.00 